\title{
Effect of acetaminophen and fluvastatin on post-dose symptoms following infusion of zoledronic acid
}

\author{
S. L. Silverman • A. Kriegman • J. Goncalves • \\ F. Kianifard • T. Carlson $\cdot$ E. Leary
}

Received: 20 May 2010/Accepted: 29 September 2010 /Published online: 30 November 2010

(C) The Author(s) 2010. This article is published with open access at Springerlink.com

\begin{abstract}
Summary A randomized, double-blind, placebo-controlled study assessed the efficacy of acetaminophen or fluvastatin in preventing post-dose symptoms (increases in body temperature or use of rescue medication) following a single infusion of the intravenous (IV) bisphosphonate zoledronic acid (ZOL). Acetaminophen, but not fluvastatin, significantly reduced the incidence and severity of post-dose symptoms. Introduction Transient symptoms including myalgia and pyrexia have been reported post-infusion of IV bisphosphonates, typically starting the day after infusion and resolving within several days. The cause is unknown but may be related to transient cytokine elevations. Statins' potential to block release of these cytokines has been hypothesized. This study was aimed to evaluate efficacy of acetaminophen and fluvastatin in preventing/reducing post-dose symptoms following ZOL $5 \mathrm{mg}$ infusion.

Methods Randomized, double-blind, placebo-controlled study of efficacy of acetaminophen or fluvastatin in
\end{abstract}

S. L. Silverman

Cedars-Sinai Medical Center and David Geffen School

of Medicine, University of California,

Los Angeles, CA, USA

\author{
A. Kriegman · J. Goncalves $\cdot$ F. Kianifard \\ Novartis Pharmaceuticals, \\ East Hanover, NJ, USA \\ T. Carlson $\cdot$ E. Leary \\ Pacific Biomarkers, \\ Seattle, WA, USA \\ S. L. Silverman $(\square)$ \\ Cedars-Sinai/UCLA, \\ 8641 Wilshire Blvd, Suite 301, \\ Beverly Hills, CA 90211, USA \\ e-mail: stuarts@omcresearch.org
}

preventing increases in body temperature or use of rescue medication (ibuprofen) following a single ZOL infusion. Bisphosphonate-naive postmenopausal women with low bone mass $(N=793)$ were randomized into three treatment groups and given $650 \mathrm{mg}$ acetaminophen or $80 \mathrm{mg}$ fluvastatin or placebo $45 \mathrm{~min}$ before ZOL infusion. The acetaminophen group continued taking $650 \mathrm{mg}$ acetaminophen every $6 \mathrm{~h}$ over the next 3 days, and the other two groups took matching placebo according to the same schedule. Subjects recorded body temperature, symptoms in a diary. Inflammatory cytokines and C-reactive protein (CRP) were measured at baseline, 24, and $72 \mathrm{~h}$ in a study subset.

Results Acetaminophen four times/day significantly reduced the incidence and severity of post-dose symptoms following ZOL infusion. Single-dose fluvastatin $80 \mathrm{mg}$ prior to ZOL infusion did not prevent/reduce post-dose symptoms. Cytokine levels increased by $24 \mathrm{~h}$ and returned towards baseline by $72 \mathrm{~h}$, similar to the pattern for post-infusion symptoms. CRP levels increased from baseline to $72 \mathrm{~h}$. Conclusions Acetaminophen four times/day for 3 days significantly reduced the incidence and severity of post-dose symptoms following ZOL infusion.

Keywords Cytokines · Fluvastatin · Inflammatory biomarkers · Osteoporosis · Post-dose symptoms . Zoledronic acid

\section{Introduction}

Zoledronic acid (ZOL) is a nitrogen-containing intravenous (IV) bisphosphonate that is approved for the treatment and prevention of postmenopausal osteoporosis, for increasing bone mass in men with osteoporosis, and for treatment and 
prevention of glucocorticoid-induced osteoporosis. In a placebo-controlled clinical trial of postmenopausal women with osteoporosis (Health Outcomes and Reduced Incidence with Zoledronic Acid Once Yearly [HORIZON] - Pivotal Fracture Trial), once-yearly infusions of ZOL $5 \mathrm{mg}$ significantly reduced the risk of morphometric vertebral fracture by $70 \%(p<0.0001)$ and of hip fracture by $41 \%(p=0.002)$ over 36 months [1]. A subsequent placebo-controlled trial (HORIZON-Recurrent Fracture Trial) demonstrated that an annual infusion of ZOL after a recent low-trauma hip fracture significantly reduced the incidence of clinical fractures by $35 \%(p=0.001)$ compared with placebo [2].

The most common adverse events (AEs) associated with $\mathrm{ZOL}$ are transient post-dose symptoms (also referred to as an acute phase response) consisting of fever, myalgia, arthralgia, headache, and flu-like symptoms [1]. These symptoms may occur following initial treatment with IV bisphosphonates; however, the incidence decreases substantially with subsequent treatments $[1,3]$. In the HORIZON-Pivotal Fracture Trial, the proportion of patients experiencing any of the five most common post-dose symptoms decreased from $32 \%$ after the first dose to $7 \%$ after the second annual dose and to $3 \%$ after the third annual dose [1]. Post-dose symptoms are generally mild to moderate in severity, and most resolve within 3 days, but some may last for 7-14 days [3, 4]. Treatment with analgesics has been reported to mitigate symptoms [3].

The mechanism for the acute phase response appears to be associated with the transient release of inflammatory cytokines (e.g., interleukin-6 [IL-6], tumor necrosis factor alpha $[\mathrm{TNF}-\alpha]$ ) from gamma delta T-cells [5, 6]. Isopentenyl pyrophosphate (IPP) is a known potent activator of gamma delta T-cells [7-9]. ZOL inhibits osteoclast-mediated bone resorption by blocking farnesyl pyrophosphate synthase (FPS), a key enzyme in the mevalonate pathway [10, 11]. Blockade of FPS, in turn, results in increased levels of IPP in monocytes [7]. It is believed that the acute phase response following ZOL infusion occurs as a result of IPP-induced Tcell activation and the subsequent release of inflammatory mediators.

Statins are commonly used cholesterol-lowering drugs that act by inhibiting 3-hydroxy-3-methyl-glutaryl-coenzyme A (HMG-CoA) reductase, a precursor of IPP and cholesterol in the mevalonate pathway. Inhibition of HMG-CoA reductase therefore prevents the synthesis of IPP. In vitro, co-treatment of blood mononuclear cells with a statin and a nitrogencontaining bisphosphonate (N-BP) completely prevents proliferation and activation of gamma delta T-cells caused by N-BPs [12]. We therefore hypothesized that co-administration of a statin with ZOL would have the potential to reduce IPP accumulation in monocytes, prevent proliferation and activation of gamma delta T-cells, and decrease the subsequent acute phase response.
ZOL is infused over $15 \mathrm{~min}$ and then rapidly binds to bone. Any drug that does not bind to bone is excreted by the kidney within $24 \mathrm{~h}$. Therefore, a sufficient blood level of statin would need to be present in the circulation prior to ZOL administration and during the subsequent $24 \mathrm{~h}$ in order to determine whether the acute phase response could be attenuated in humans.

Fluvastatin $80 \mathrm{mg}$ immediate release formulation was chosen as the statin regimen for this study because this dose was approved for another indication (cholesterol-lowering) and pharmacokinetic data indicated that the immediate release formulation would provide high, rapid levels of circulating drug. Fluvastatin was dosed approximately 45 min prior to ZOL infusion in order to allow time for oral absorption and peak blood levels of fluvastatin at the time of ZOL infusion. No additional doses of fluvastatin were given in this study.

Here, we report findings from a randomized, doubleblind study that compared the effects of acetaminophen, fluvastatin, and placebo on transient post-dose symptoms and inflammatory biomarker levels following a single dose of ZOL in postmenopausal women with low bone mass. Our hypothesis was that both acetaminophen and fluvastatin would reduce the incidence and severity of post-dose symptoms - the former, based on its antipyretic and analgesic properties, and the latter, based on the potential for inhibition of cytokine release (as suggested by in vitro data [12]). We further hypothesized that reduction in post-dose symptoms would be linked with reductions in the levels of inflammatory biomarkers.

\section{Methods}

\section{Study design}

We conducted a randomized, multicenter, double-blind, placebo-controlled, double-dummy, parallel group study to evaluate the efficacy and safety of acetaminophen or fluvastatin (Lescol; $\left.R^{*}, S^{*}-(E)\right]-( \pm)$-7-[3-(4-fluorophenyl)-1(1-methylethyl)-1 $H$-indol-2-yl]-3,5-dihydroxy-6-heptenoic acid, monosodium salt; Novartis Pharma) in preventing clinically significant increases in body temperature or use of rescue medication (ibuprofen) following a single infusion of ZOL (Reclast; [1-Hydroxy-2-imidazol-1-ylphosphonoethyl] phosphonic acid monohydrate; Novartis Pharma). The study was conducted at 94 sites in the USA between June and December 2007. It was approved by appropriate institutional review boards and conducted according to the International Conference on Harmonisation of Technical Requirements for Registration of Pharmaceuticals for Human Use, local guidelines, and the ethical principles of the Declaration of Helsinki. 
Informed consent was obtained from each patient prior to conducting any study procedures.

The study included a screening visit and a screening period of up to 31 days, followed by a randomization/ infusion visit (Day 1), a 3-day treatment period, and a final visit (14 to 21 days after the infusion). Patients were given a bottle of tablets containing calcium $(600 \mathrm{mg})$ and vitamin D3 (400 mg) at the screening visit and were instructed to take two tablets daily for the duration of the study. During the screening period, patients were instructed to stop taking acetaminophen, nonsteroidal anti-inflammatory drugs (NSAIDs), glucocorticosteroids, and statin medications to comply with the required washout interval.

Body temperature was measured orally at baseline. Patients completed a symptom questionnaire and recorded severity of baseline symptoms on a 100 -mm visual analog scale (VAS; 0 [no symptoms] to 100 [severe symptoms]). The symptom questionnaire consisted of three questions (severity of fever, severity of headache, and severity of aches and pains), each rated on a four-point categorical scale (0 [absent] to three [severe]). Study medication (acetaminophen, fluvastatin, or placebo) was administered $45 \pm 15 \mathrm{~min}$ before ZOL administration. Rescue medication (unblinded ibuprofen) was dispensed, and patients were instructed to take ibuprofen in addition to study medication if they experienced severe discomfort. During the 3-day treatment period, patients completed the symptom questionnaire four times daily (morning, midday, evening, and late evening) and then recorded their oral body temperature prior to taking study medication (acetaminophen/matching placebo). The VAS score was recorded once per day in the late evening. At the final visit, patient diaries were collected and patients returned used bottles and unused study and rescue medication. AEs and clinical chemistry variables were evaluated.

Patients in the exploratory inflammatory biomarker subgroup had their first blood sample drawn on Day 1 prior to ingesting their blinded study medications. Additional blood samples were collected at $24 \pm 2$ and $72 \pm 2 \mathrm{~h}$ after ZOL infusion. Blood samples were processed by Quintiles Transnational (Durham, NC), and highly sensitive serum biomarker assays capable of measuring low normal levels were performed by Pacific Biomarkers of Seattle, WA (IL-6 and TNF-alpha: R\&D Systems, Minneapolis, $\mathrm{MN}$; interferon [IFN]-gamma: Meso Scale Discovery, Gaithersburg, MD; highly sensitive CRP [hs-CRP]: Roche Diagnostics North America, Indianapolis, IN).

The primary objective of this study was to demonstrate the superiority of acetaminophen vs. placebo in preventing clinically significant increases in body temperature or use of rescue medication during 3 days following $\mathrm{ZOL}$ infusion. Secondary objectives included assessment of whether fluvastatin was superior to placebo in preventing clinically significant increases in body temperature measured orally or use of rescue medication.

\section{Patients}

Postmenopausal women aged between 45 and 79 years with a clinical indication for bisphosphonate treatment for osteopenia or osteoporosis and a documented spine or hip bone mineral density dual-energy X-ray absorptiometry $T$-score of -1.5 or less were eligible for participation in this study. Women who had used IV bisphosphonates or taken oral bisphosphonates for more than 8 weeks or within 6 months of screening were excluded. Other exclusion criteria included the use of 3-hydroxy-3-methylglutarylcoenzyme A (HMG-CoA) reductase inhibitors (statins) or systemic IV or oral corticosteroids within 7 days prior to randomization; the use of acetaminophen, NSAIDs, tranquilizers, muscle relaxants, raloxifene, or calcitonin within $36 \mathrm{~h}$ of randomization; or the use of anticoagulant therapy. Patients with proteinuria (urine dipstick $\geq 2+$ ), impaired creatinine clearance $(<30 \mathrm{ml} / \mathrm{min})$, or abnormal serum calcium levels $(>2.75$ or $<2.08 \mathrm{mmol} / \mathrm{l})$ at screening were not eligible for study participation. Patients with an ongoing infection (including dental infection) or planned oral surgery within 3 months of randomization were also excluded.

\section{Study medications}

All patients received an infusion of ZOL $5 \mathrm{mg}$ over at least $15 \mathrm{~min}$ on Day 1. Patients were randomized to one of three treatment groups. All oral study drugs were double-blinded with matching placebo capsules. In Group 1, 45 min prior to ZOL infusion, two capsules of acetaminophen $325 \mathrm{mg}$ were administered orally. Subjects in this group continued to take two capsules of acetaminophen four times daily for the next 3 days at home. In Group 2, 45 min prior to ZOL infusion, two capsules of immediate-release fluvastatin $40 \mathrm{mg}$ were administered orally. Fluvastatin was administered only once, on Day 1, prior to ZOL infusion. Subjects in the fluvastatin group were provided with placebo capsules (matching acetaminophen) and took two capsules four times daily for the next 3 days at home. In Group 3, subjects received placebo $45 \mathrm{~min}$ prior to $\mathrm{ZOL}$ infusion and continued to take two capsules of placebo (matching acetaminophen) four times daily for the next 3 days at home.

Patients in this study were also provided with calcium $600 \mathrm{mg}$ plus vitamin D3 400 IU (one tablet twice daily). Open-label rescue medication (ibuprofen $200 \mathrm{mg}$ tablets every 4 to $6 \mathrm{~h}$, not to exceed eight tablets in a 24-h period) could be taken if the patient had an oral body temperature $\geq 38.9^{\circ} \mathrm{C}$ 
$\left(102^{\circ} \mathrm{F}\right)$ and/or severe symptoms of fever, headache, myalgia, or arthralgia.

Study personnel observed each patient ingest the first dose of study medication and receive the ZOL infusion. Patient diaries and unused medication were used to assess compliance during the remainder of the study. Patients recorded the dose, date, and time of study and rescue medication use in diaries and returned used bottles and unused study and rescue medication at the final visit.

\section{Efficacy and safety variables}

The primary efficacy variable in this study was the proportion of patients who had a clinically significant increase in oral body temperature $\left(\geq 1^{\circ} \mathrm{C}\right.$ from baseline and $\geq 38.5^{\circ} \mathrm{C}$ overall) or used rescue medication at least once during the 3-day period following ZOL infusion. Oral body temperature was measured at baseline prior to ZOL infusion using a digital thermometer (Welch Allyn Sure Temp), which was provided to each patient for the duration of the study. Patients were trained to take two temperature assessments within $10 \mathrm{~min}$ of each other four times per day for 3 days. Temperature assessments were conducted after completing VAS and symptom questionnaires and prior to taking any oral study medication. Patients recorded the time and number of tablets of rescue medication taken, if any, in their diaries.

Secondary efficacy variables included the proportion of patients with a clinically significant increase in body temperature and the proportion of patients who used rescue medication. Change from baseline in mean temperature, change from baseline in symptom VAS, major increases in severity of symptoms (an increase from baseline of a minimum of two units on the symptom questionnaire at least once during the 3 days immediately following ZOL infusion), and severe symptoms (reported at least once) were also examined. Levels of inflammatory biomarkers (IL-6, TNF-alpha, IFN-gamma, hs-CRP) in a subgroup of patients were exploratory variables.

AEs were monitored and recorded throughout the study. Physical examinations and evaluations of vital signs and clinical chemistry were performed at the screening and final visits.

\section{Statistical analyses}

Statistical analyses were performed by Rho (Cary, NC) using SAS statistical software (version 9.1). Assuming that the proportion of patients with a clinically significant increase in oral body temperature was 33\% in the placebo group and $19 \%$ in the acetaminophen group and that the dropout rate was $10 \%$, the study would require 243 patients per group (total of 729 patients) to have at least $90 \%$ power to detect a difference between the two groups. This calculation used a two-group continuity-corrected Chisquare test with a two-sided significance level of 0.05 .

The primary efficacy variable (clinically significant increase in temperature or rescue medication) was analyzed using a logistic regression model with treatment and baseline oral body temperature (mean of two temperatures recorded at baseline) as explanatory variables; odds ratios (OR) for pairwise treatment comparisons, 95\% confidence intervals (CI) for $\mathrm{OR}$, and $p$ values are presented. Two binary secondary efficacy variables (clinically significant increase in temperature, rescue medication use) were similarly analyzed.

Change from baseline in symptom VAS was analyzed by an analysis of covariance model with treatment and baseline VAS as explanatory variables. Between-treatment comparisons of proportions of patients with major increases in severity of symptoms and severe symptoms (reported at least once) were made based on pairwise Chi-square tests.

Correlations between changes in inflammatory biomarkers and changes in temperature or symptoms were evaluated by use of Pearson and Spearman correlation coefficients.

\section{Results}

\section{Patients}

Of 1,008 patients screened, 793 were randomized, and 779 completed the study. All analyses were conducted on the 793 randomized patients. The primary reason for withdrawal was AEs (ten of 14 withdrawals). Overall withdrawals and withdrawals due to AEs occurred at comparable rates in the three treatment groups. Treatment groups were generally well matched with respect to baseline characteristics. Overall, $90.5 \%$ of the study population was Caucasian, the mean age was 61.7 years, and the mean number of menopausal years was 15.7. In the subgroup of patients with inflammatory biomarker data ( $n=96$, placebo 33 patients, acetaminophen 33 patients, fluvastatin 30 patients), demographic and background characteristics were similar to those in the ITT population, and the treatment groups remained well matched. Compliance was excellent and well balanced across treatment groups. There were no compliance issues with respect to fluvastatin, as the sole dose was administered by study personnel; for acetaminophen and acetaminophen-matching placebo, the mean number of capsules taken ranged from 21.2 to 21.5 (out of 24 ).

\section{Efficacy outcomes}

Following a single infusion of ZOL $5 \mathrm{mg}$, acetaminophen was found to be superior to placebo in preventing or reducing post-dose symptoms over the subsequent 3-day 


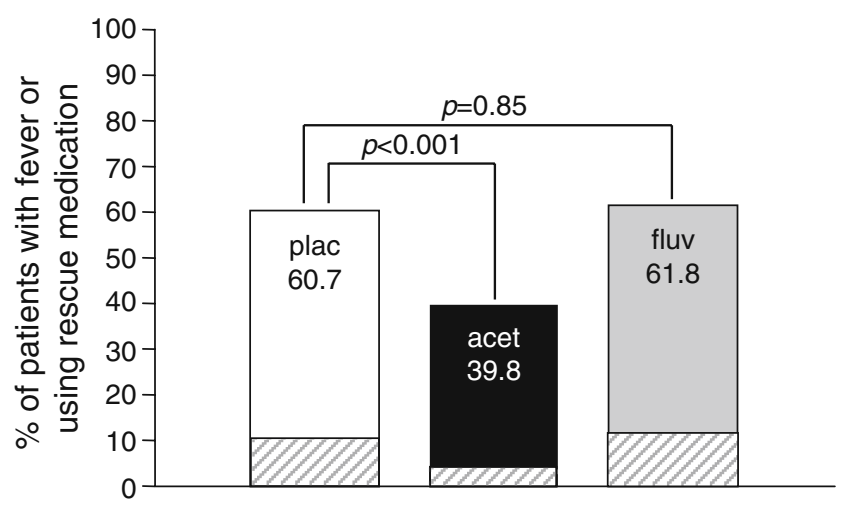

Fig. 1 Proportion of patients with fever (clinically significant increase in oral body temperature $\left[1^{\circ} \mathrm{C}\right.$ or more from baseline and $38.5^{\circ} \mathrm{C}$ or more overall]) or use of at least one dose of rescue medication during the 3-day period following IV zoledronic acid infusion (intent-to-treat population). Cross-hatching indicates proportion of patients in each group with one or more episodes of fever recorded in the patient diary (no $p$ values shown for the latter comparisons). acet acetaminophen, fluv fluvastatin, plac placebo

period. Clinically significant increases in oral body temperature or use of rescue medication occurred in $60.7 \%$ (162) of 267 patients in the placebo group vs. $39.8 \%(105$ of 264 patients) in the acetaminophen group $(p<0.001$; Fig. 1). In contrast, no effect was observed from a single dose of fluvastatin taken 45 min prior to the ZOL infusion, with a total of $61.8 \%$ of patients (162 of 262) having increased temperature or using rescue medication. Subgroup analyses showed that all age groups (45-55 years, 56-64 years, and 65 years and older) experienced significant benefits from acetaminophen.
Acetaminophen also produced significant benefits with respect to secondary efficacy variables. Compared with placebo, acetaminophen significantly decreased the proportion of patients with increased body temperature, of those who used rescue medication, of those who experienced a major increase in severity of symptoms, and of those who reported at least one episode of severe symptoms. Fluvastatin did not significantly affect any symptom variables (Table 1).

Compared with patients in the fluvastatin and placebo groups, patients in the acetaminophen group had a lower peak increase in body temperature and an earlier return to baseline levels (Fig. 2a). For each treatment group, the largest mean increase in temperature occurred between 24 and $48 \mathrm{~h}$ following ZOL infusion, and the peak value was recorded at the Day 2 evening measurement. The symptom VAS (recorded once each evening) followed a similar pattern (Fig. 2b), with peak values on Day 2, and the mean difference between placebo and acetaminophen was statistically significant at all time points $(p<0.05)$. In contrast, no significant differences were observed between placebo and fluvastatin.

\section{Inflammatory biomarkers}

Serum levels of inflammatory biomarkers were evaluated in 96 patients at baseline, $24 \mathrm{~h}$, and $72 \mathrm{~h}$. Baseline concentrations of IL-6, IFN-gamma, TNF-alpha, and hs-CRP were generally comparable across treatment groups (Table 2). The pattern of elevations of all four inflammatory biomarkers showed an increase in levels by $24 \mathrm{~h}$ after infusion (Day 2, morning; Table 2; Fig. 3a-d); elevations in body

Table 1 Clinically significant increase in oral body temperature, rescue medication use, worsening symptoms, and severe symptoms during the 3-day period following IV zoledronic acid infusion

\begin{tabular}{|c|c|c|c|c|c|c|}
\hline \multirow[t]{2}{*}{ Variables } & \multicolumn{2}{|c|}{ PLAC $(N=267)$} & \multicolumn{2}{|c|}{$\operatorname{ACET}(N=264)$} & \multicolumn{2}{|c|}{ FLUV $(N=262)$} \\
\hline & Number & Percentage $(\%)$ & Number & Percentage $(\%)$ & Number & Percentage $(\%)$ \\
\hline Clinically significant increase in temperature ${ }^{a}$ & 28 & 10.5 & 13 & $4.9^{\mathrm{b}}$ & 30 & 11.5 \\
\hline Use of rescue medication & 153 & 57.3 & 102 & $38.6^{\mathrm{c}}$ & 156 & 59.5 \\
\hline \multicolumn{7}{|l|}{ Major increase in severity of symptoms } \\
\hline Feeling feverish & 105 & 39.3 & 62 & $23.5^{\mathrm{c}}$ & 104 & 39.7 \\
\hline Headache & 104 & 39.0 & 67 & $25.4^{\mathrm{c}}$ & 115 & 43.9 \\
\hline Aches and pains & 127 & 47.6 & 89 & $33.7^{\mathrm{c}}$ & 121 & 46.2 \\
\hline \multicolumn{7}{|l|}{ Severe symptoms (reported at least once) } \\
\hline Feeling feverish & 36 & 13.5 & 20 & $7.6^{\mathrm{b}}$ & 38 & 14.5 \\
\hline Headache & 42 & 15.7 & 18 & $6.8^{\mathrm{c}}$ & 42 & 16.0 \\
\hline Aches and pains & 81 & 30.3 & 56 & $21.2^{\mathrm{b}}$ & 79 & 30.2 \\
\hline
\end{tabular}

ACET acetaminophen, FLUV fluvastatin, PLAC placebo

${ }^{\mathrm{a}} 1^{\circ} \mathrm{C}$ or more from baseline and $38.5^{\circ} \mathrm{C}$ or more overall

${ }^{\mathrm{b}} p<0.05$ vs. placebo

${ }^{\mathrm{c}} p \leq 0.001$ vs. placebo 


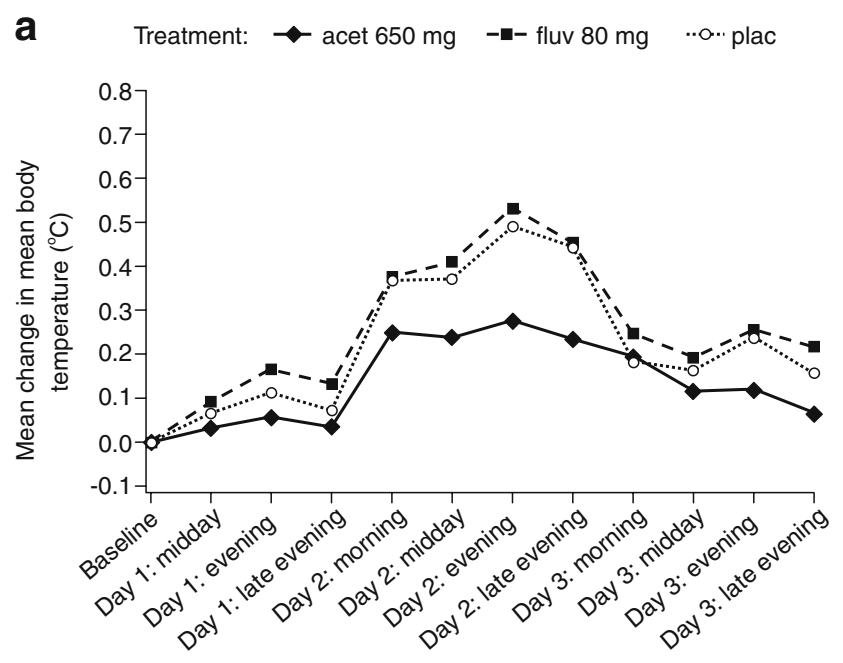

b Treatment: $\multimap$ acet $650 \mathrm{mg} \quad$-!- fluv $80 \mathrm{mg} \quad$..o.. plac

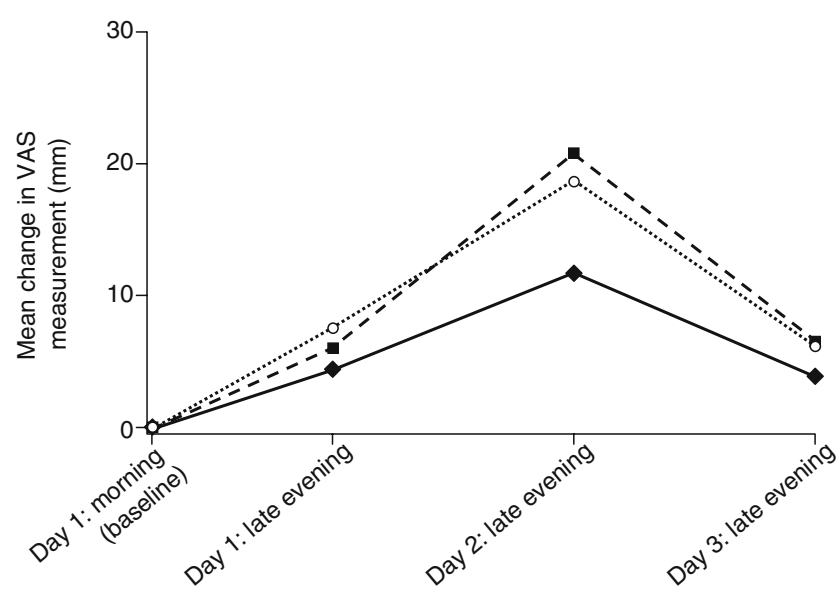

Fig. 2 Change from baseline in a mean body temperature and $\mathbf{b}$ VAS scores following IV zoledronic acid infusion in patients who received pretreatment with fluvastatin $(f l u v)$, acetaminophen four times daily over 3 days (acet), or placebo (plac)

temperature were also reported on the morning of Day 2 (Fig. 2a). Levels of all three cytokines (IL-6, TNF-alpha, and IFN-gamma) returned to near baseline by $72 \mathrm{~h}$, by which point most of the temperature elevations had declined. The biomarker, CRP, continued to rise from baseline to $72 \mathrm{~h}$.

Inflammatory biomarker changes were weakly correlated with changes in body temperature and VAS scores. IL-6, IFN-gamma, and hs-CRP levels were generally higher in patients with a major increase in symptom severity (with the exception of severe headaches). However, some asymptomatic patients also experienced biomarker elevations. The use of acetaminophen appeared to attenuate increases in IL-6 and IFN-gamma levels at $24 \mathrm{~h}$ in this treatment group compared with those reported for the placebo and fluvastatin groups (Fig. 3a, c).
Safety

In safety evaluations, post-dose symptoms were not counted as AEs, since they were collected in patient diaries as secondary outcomes. The most common AEs were musculoskeletal and connective tissue disorders, general disorders and administration site conditions, and gastrointestinal disorders. AEs occurred at comparable rates across treatment groups.

There were no deaths in the study. Five $(0.6 \%)$ patients reported six serious AEs (one [hypokalemia] in the placebo group, two [syncope and pleuritic pain] in the acetaminophen group, and three [convulsion, pyrexia, and uveitis] in the fluvastatin group). Ten (1.3\%) patients withdrew from the study due to AEs (three in the placebo group, three in the acetaminophen group, and four in the fluvastatin group). There were no notable differences between treatment groups in serious AEs or treatment withdrawals. No clinically significant between-group differences were observed in laboratory values or vital signs.

\section{Discussion}

Transient post-dose symptoms are the most frequently reported AEs following ZOL infusions in postmenopausal

Table 2 Serum levels of inflammatory biomarkers

\begin{tabular}{|c|c|c|c|}
\hline & $\operatorname{PLAC}(N=33)$ & $\operatorname{ACET}(N=33)$ & $\operatorname{FLUV}(N=30)$ \\
\hline \multicolumn{4}{|c|}{ IL-6 (pg/ml): median (min, max $)^{\mathrm{a}}$} \\
\hline Baseline & $2.0(1,61)$ & $2.1(0,31)$ & $2.5(0,8)$ \\
\hline $24 \mathrm{~h}$ & $14.5(2,154)$ & $9.7(2,73)$ & $14.8(2,79)$ \\
\hline $72 \mathrm{~h}$ & $3.9(1,160)$ & $2.8(1,56)$ & $3.5(2,79)$ \\
\hline \multicolumn{4}{|c|}{ TNF-alpha (pg/ml): median $(\min , \max )^{\mathrm{b}}$} \\
\hline Baseline & $1.9(1,5)$ & $1.9(1,9)$ & $1.8(1,7)$ \\
\hline $24 \mathrm{~h}$ & $3.8(1,9)$ & $3.7(2,11)$ & $4.1(2,12)$ \\
\hline $72 \mathrm{~h}$ & $2.6(1,7)$ & $2.2(0,12)$ & $3.8(1,9)$ \\
\hline \multicolumn{4}{|c|}{ IFN-gamma $(\mathrm{pg} / \mathrm{ml}):$ median $(\min , \max )^{\mathrm{c}}$} \\
\hline Baseline & $0.6(1,4)$ & $0.6(1,4)$ & $0.6(1,2)$ \\
\hline $24 \mathrm{~h}$ & $75.5(1,363)$ & $40.7(1,872)$ & $98.2(4,3479)$ \\
\hline $72 \mathrm{~h}$ & $2.0(1,24)$ & $1.6(1,12)$ & $3.1(1,10)$ \\
\hline \multicolumn{4}{|c|}{ hs-CRP (mg/l): median $(\min , \max )^{d}$} \\
\hline Baseline & $2.3(0,13)$ & $2.3(1,8)$ & $1.8(0,49)$ \\
\hline $24 \mathrm{~h}$ & $8.0(0,81)$ & $4.7(1,45)$ & $7.8(0,77)$ \\
\hline $72 \mathrm{~h}$ & $25.1(0,89)$ & $19.3(1,133)$ & $20.2(0,71)$ \\
\hline
\end{tabular}

$A C E T$ acetaminophen, FLUV fluvastatin, $h s-C R P$ highly sensitive Creactive protein, $P L A C$ placebo

${ }^{\mathrm{a}} \mathrm{IL}-6$ (pg/ml) normal reference range: 0.51-4.92

${ }^{\mathrm{b}}$ TNF-alpha (pg/ml) normal reference range: less than 1.86

${ }^{\mathrm{c}}$ IFN-gamma (pg/ml) normal reference range: less than 1.9

${ }^{\mathrm{d}}$ hs-CRP (mg/l) normal reference range: less than 3.0 
Fig. 3 Change from baseline in serum levels of a IL-6, b TNF-alpha, c IFN-gamma, and $\mathbf{d}$ hs-CRP following IV zoledronic acid infusion in a subset of 96 patients receiving treatment with placebo (plac), acetaminophen (acet), or fluvastatin $(f l u v)$. Measurements were taken at baseline and at 24 and $72 \mathrm{~h}$ post-infusion. $h s$-CRP highly sensitive C-reactive protein, $I F N$ interferon, $I L$ interleukin, $T N F$ tumor necrosis factor a

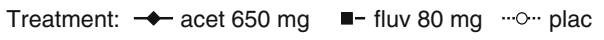

C

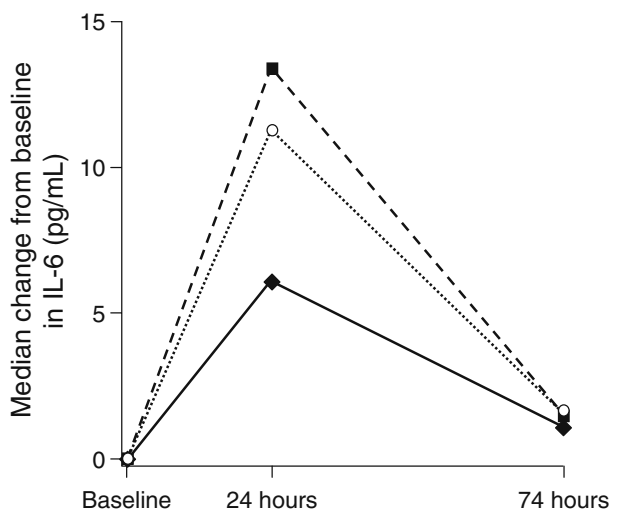

b
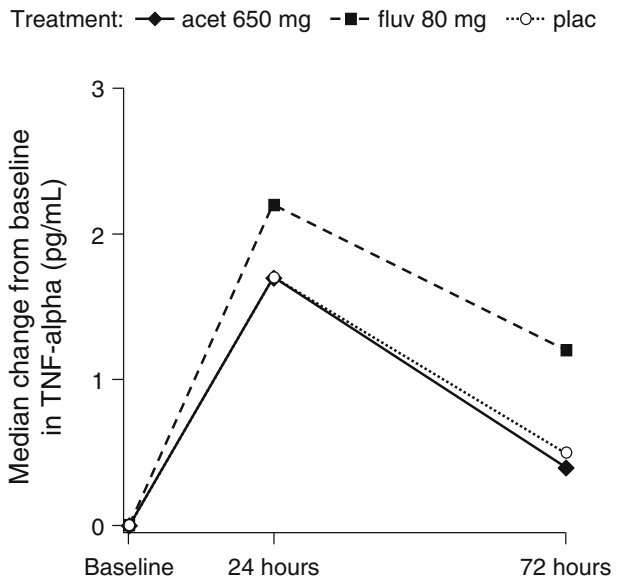
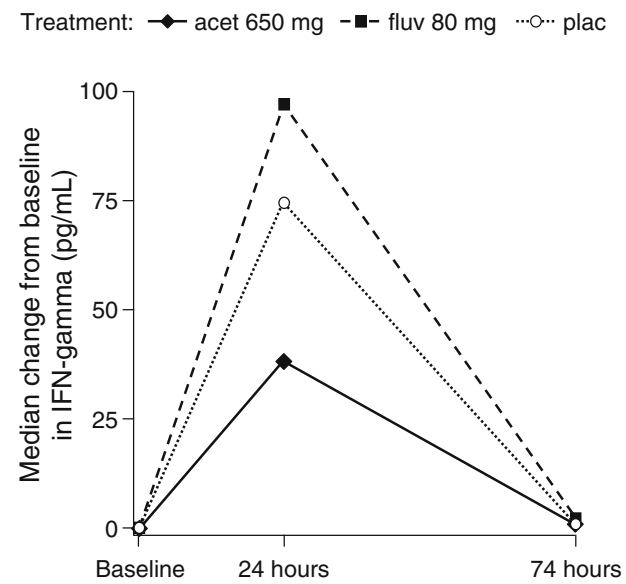

d
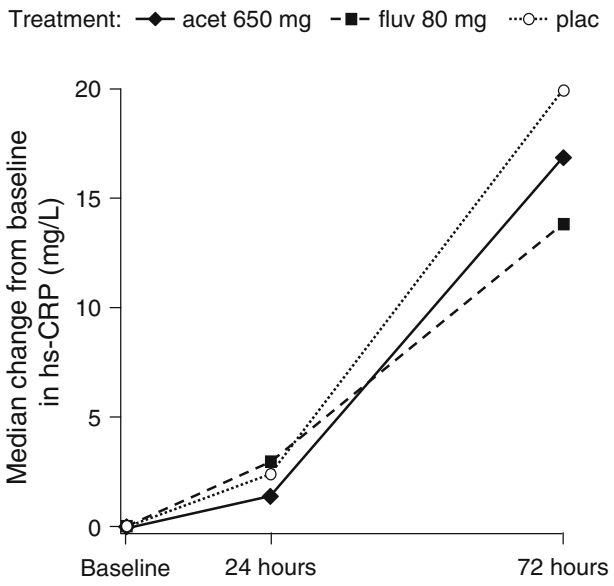

women [1]. These symptoms are believed to be caused by the accumulation of IPP as a result of FPP blockade in the mevalonate pathway, a key step in the inhibition of bone resorption by ZOL [10]. Statins block an earlier stage of this pathway and do not lead to IPP accumulation. In vitro, pretreatment of peripheral blood mononuclear cells with a statin followed by exposure of the cells to a bisphosphonate prevents the bisphosphonate-induced release of inflammatory cytokines including TNF-alpha and IFN-gamma [12].

In this trial, we evaluated the efficacy of acetaminophen and fluvastatin in preventing or reducing post-dose symptoms following administration of a single infusion of $\mathrm{ZOL}$ in bisphosphonate-naive postmenopausal women with low bone mass. The primary objective of this study was met: acetaminophen was significantly more effective than placebo in reducing clinically significant increases in body temperature or use of rescue medication. Acetaminophen resulted in substantial reductions in the incidence and severity of symptoms and was effective in all age groups. In contrast, pretreatment with a single dose of immediaterelease fluvastatin given prior to $\mathrm{ZOL}$ infusion failed to demonstrate a significant effect on post-dose symptoms in any of the analyses conducted.

Exploratory analyses of inflammatory biomarkers in a subset of patients provided insights into potential mechanisms for the manifestation of post-dose symptoms. The timing of the maximum increases in levels of IL-6, TNF-alpha, and IFN-gamma were generally similar to the timing of the maximum increases in body temperature and VAS scores (Figs. 2 and 3), with elevations occurring between baseline and $24 \mathrm{~h}$ and levels returning to near baseline by $72 \mathrm{~h}$. Changes in CRP showed a different pattern, with levels continuing to increase between 24 and $72 \mathrm{~h}$. However, it should be noted that CRP synthesis is upregulated by inflammatory cytokines, including IL-6. Serum CRP levels begin to increase as soon as the inflammatory stimuli ebb and therefore may exhibit a later increase and slower decline than cytokine levels [13].

IL-6, IFN-gamma, and CRP levels were generally higher in patients with a major increase in symptom severity (with the exception of severe headaches). However, both asymptomatic and symptomatic patients experienced biomarker 
elevations, so the correlation between symptom severity and biomarker levels was weak. Acetaminophen, but not fluvastatin, attenuated increases in IL-6 and IFN-gamma levels compared with placebo following ZOL infusion.

In this study, $39.3 \%$ of placebo-treated patients reported a major increase in feeling feverish over the 3-day treatment period (Table 1), compared with $9 \%-16 \%$ of patients in previous ZOL trials who spontaneously reported post-infusion fever symptoms at the next office visit $[1,2]$. In terms of objective temperature measurements, $10.5 \%$ of placebo patients in the current study experienced at least one clinically significant elevation in oral body temperature (similar to the percentage spontaneously reporting fever in previous ZOL trials); however, $57.3 \%$ of patients took at least one dose of ibuprofen, which may have lowered the maximum temperature increase. Regarding cytokine levels, our findings are in partial agreement with other studies examining cytokine profiles following IV bisphosphonate infusions. As in the studies by Thiébaud et al. [5] and Dicuonzo et al. [6], we found that the pattern of IL- 6 elevations closely mirrored the time course of post-dose symptoms and that IL- 6 increases were greater in patients with symptoms. However, our data support a potential role for IFN-gamma in mediating postdose symptoms, whereas the study by Dicuonzo and colleagues [6] did not. Differences in study populations or use of more sensitive biomarker assays in our study may help to explain this discrepancy.

CRP levels were increased at $72 \mathrm{~h}$ in the current study, and no additional measurements were available at later time points. However, data from a study by Michael Rogers and colleagues showed that elevations in CRP levels after a ZOL 5-mg infusion were back to baseline levels when measured 4 weeks post-infusion (Keith Thompson and Michael Rogers, personal communication).

Although pretreatment with statins has been shown to block bisphosphonate-induced cytokine release in vitro [12], this clinical study did not demonstrate any benefit of dosing with fluvastatin prior to ZOL infusion. Our findings are consistent with those of a recent study by Srivastava and colleagues [14] in which atorvastatin $10 \mathrm{mg}$ was administered to children with metabolic bone diseases receiving IV bisphosphonate treatment. Atorvastatin did not result in significant reductions in pain, rescue medication use, or CRP levels, leading the authors to conclude that this agent was not effective in modulating bisphosphonate-induced post-dose responses. Data from clinical studies thus suggest that statins do not reduce the incidence of post-infusion symptoms.

Our study implicates IL-6 and IFN-gamma in the induction of post-dose symptoms, as both biomarkers showed marked elevations following ZOL infusion and their temporal patterns closely mirrored changes in body temperature and VAS symptom scores. In addition, acet- aminophen reduced symptom scores and resulted in lower peak levels of these cytokines at $24 \mathrm{~h}$.

Limitations of the current study include the 72-h duration of inflammatory biomarker monitoring; additional data after $72 \mathrm{~h}$ may have been useful to document ongoing changes in CRP and determine when levels returned to baseline values. Moreover, we did not know the optimal dose of fluvastatin, or the optimal timing of its administration for use in this setting.

We conclude that acetaminophen is effective in significantly reducing the incidence and severity of post-dose symptoms following ZOL infusion. Exploratory analyses of inflammatory biomarkers suggest that acetaminophenmediated reductions in IL- 6 and IFN-gamma levels may help to explain the effect of this agent on post-dose symptoms. In contrast to acetaminophen, pretreatment with a single dose of fluvastatin did not show any benefit in mitigating post-dose symptoms. Based on these data, we encourage clinicians to consider the use of acetaminophen $650 \mathrm{mg}$ four times daily for 3 days for the reduction of post-dose symptoms following ZOL infusions.

Acknowledgments The authors wish to thank the investigators at the various trial sites for their efforts, Neepa Ray of Rho for statistical programming, and Eric Justice of BioScience Communications (New York, NY) for editorial assistance in the development of this manuscript which is funded by Novartis Pharmaceuticals (East Hanover, NJ).

Conflicts of interest This study and the writing of this manuscript were funded by Novartis Pharmaceuticals (East Hanover, NJ). Dr. Silverman has served as a consultant for Novartis on speakers' bureaus and advisory boards. Drs. Kriegman, Goncalves, and Kianifard are employees of Novartis. Drs. Carlson and Leary are employees of Pacific Biomarkers (Seattle, WA).

Open Access This article is distributed under the terms of the Creative Commons Attribution Noncommercial License which permits any noncommercial use, distribution, and reproduction in any medium, provided the original author(s) and source are credited.

\section{References}

1. Black DM, Delmas PD, Eastell R et al (2007) Once-yearly zoledronic acid for treatment of postmenopausal osteoporosis. N Engl J Med 356:1809-1822

2. Lyles KW, Colón-Emeric CS, Magaziner JS et al (2007) Zoledronic acid and clinical fractures and mortality after hip fracture. N Engl J Med 357:1799-1809

3. Tanvetyanon T, Stiff PJ (2006) Management of the adverse effects associated with intravenous bisphosphonates. Ann Oncol 17:897-907

4. Reclast ${ }^{\circledR}$ (zoledronic acid) prescribing information (2009) Novartis Pharmaceuticals, East Hanover, NJ

5. Thiébaud D, Sauty A, Burckhardt P et al (1997) An in vitro and in vivo study of cytokines in the acute-phase response associated with bisphosphonates. Calcif Tissue Int 61:386-392 
6. Dicuonzo G, Vincenzi B, Santini D et al (2003) Fever after zoledronic acid administration is due to increase in TNF- $\alpha$ and IL6. J Interferon Cytokine Res 23:649-654

7. Roelofs AJ, Jauhiainen M, Mönkkönen H et al (2009) Peripheral blood monocytes are responsible for $\gamma \delta \mathrm{T}$ cell activation induced by zoledronic acid through accumulation of IPP/DMAPP. Br J Haematol 144:245-250

8. Lafont V, Liautard J, Sable-Teychene M et al (2001) Isopentenyl pyrophosphate, a mycobacterial non-peptidic antigen, triggers delayed and highly sustained signaling in human gamma delta $\mathrm{T}$ lymphocytes without inducing downmodulation of $\mathrm{T}$ cell antigen receptor. J Biol Chem 276 (19): 15961-15967

9. Cipriani B, Borsellino G, Poccia F et al (2000) Activation of C-C beta-chemokines in human peripheral blood gamma delta $\mathrm{T}$ cells by isopentenyl pyrophosphate and regulation by cytokines. Blood 95(1):39-47

10. Kavanagh KL, Guo K, Dunford JE et al (2006) The molecular mechanism of nitrogen-containing bisphosphonates as antiosteoporosis drugs. Proc Natl Acad Sci USA 103:7829-7834

11. Green JR (2004) Bisphosphonates: preclinical review. Oncologist 9(Suppl 4):3-13

12. Thompson K, Rogers MJ (2004) Statins prevent bisphosphonateinduced $\gamma, \delta$-T-cell proliferation and activation in vitro. J Bone Miner Res 19:278-288

13. Pepys MB, Hirschfield GM (2003) C-reactive protein: a critical update. J Clin Invest 111:1805-1812

14. Srivastava T, Haney CJ, Alon US (2009) Atorvastatin may have no effect on acute phase reaction in children after intravenous bisphosphonate infusion. J Bone Miner Res 24:334-337 